Communications in Physics, Vol. 26, No. 3 (2016), pp. 221-228

DOI: $10.15625 / 0868-3166 / 26 / 3 / 8053$

\title{
THE 3-3-1 MODEL WITH ARBITRARILY CHARGED LEPTONS
}

\author{
HOANG NGOC LONG ${ }^{\dagger}$ \\ Institute of Physics, Vietnam Academy of Science and Technology, \\ 10 Dao Tan, Ba Dinh, Hanoi, Vietnam
}

\section{DUONG VAN LOI, NGUYEN CHI THAO}

Graduate University of Science and Technology,

Vietnam Academy of Science and Technology, 18 Hoang Quoc Viet, Cau Giay, Hanoi, Vietnam

THANH HUU HONG GIANG

Graduate School, Department of Physics, Hanoi Pedagogical University 2,

Phuc Yen, Vinh Phuc, Vietnam

${ }^{\dagger} E$-mail: hnlong@iop.vast.ac.vn

Received 27 December 2016

Accepted for publication 19 January 2017

\begin{abstract}
The gauge model based on $\mathrm{SU}(3)_{C} \otimes \mathrm{SU}(3)_{L} \otimes \mathrm{U}(1)_{X}$ group with arbitrarily electric charged exotic leptons is presented. The mass eigenvalues and eigenstates for neutral gauge bosons are presented in the general form. We show that in the 3-3-1 models, there always exists triple Higgs self-coupling. The lepton number operator is also presented.
\end{abstract}

Keywords: unified theories, models of strong, electroweak interactions.

Classification numbers: 14.60.Pq, 12.60.Cn, 14.60.St, 14.70.Pw, 12.15.Ff.

\section{INTRODUCTION}

The current status of Particle Physics shows that the Standard Model (SM) must be extended. Among beyond the Standard Models, the models based on $\mathrm{SU}(3)_{C} \otimes \mathrm{SU}(3)_{L} \otimes \mathrm{U}(1)_{X}$ (3-3-1) gauge group have some interesting properties due to the following reasons. The first reason concerns the generation number problem. In the SM, there exists the replica of the generations meaning the number of the latter is not constrained. However, in the 3-3-1 models [1,2], one of quark generation behaves differently from the two other, and this fact leads to the consequence that the number of fermion generations is multiple of the quark color number (3), i.e., $3,6, \ldots$ Combining with the Quantum Chromodynamics (QCD) asymptotic freedom requiring the quark generations is less than five, we get the answer why number of fermion generations is equal to (C)2016 Vietnam Academy of Science and Technology 
three. The second reason follows from the above mentioned fact that one of quark generations behaves differently from the two others, so we can explain why top quark is so heavy. The other issues such as neutrino and electric charge quantization [3] also get appropriate explanations.

The different kind of the 3-3-1 models is defined by the beta parameter appearing in the electric charge operator

$$
Q=T_{3}+\beta T_{8}+X
$$

In Ref. [4], the 3-3-1 models with arbitrary beta has been presented. However, this approach gives unclear content of the Higgs components. Hence, in this paper, we present the 3-3-1 model with exotic leptons having arbitrarily electric charges.

\section{THE MODEL}

In (1), $T_{i}, i=3,8$ stand for the $S U(3)_{L}$ operators. For the fundamental representation triplet

$$
T_{3}=\frac{\lambda_{3}}{2}=\frac{1}{2} \operatorname{diag}(1,-1,0), \quad T_{8}=\frac{\lambda_{8}}{2}=\frac{1}{2 \sqrt{3}} \operatorname{diag}(1,1,-2) .
$$

The lepton triplet is constituted by

$$
f_{a L}=\left(v_{a}, l_{a}, E_{a}^{q}\right)_{L}^{T} \sim(1,3,(q-1) / 3), \quad a=e, \mu, \tau,
$$

where $q$ is electric charge of associated extra lepton. Other right-handed leptons are the singlets under $S U(3)_{L}$

$$
l_{a R} \sim(1,1,-1), E_{a R}^{q} \sim(1,1, q) .
$$

Applying Eq.(1) to Eq.(2) we obtain

$$
b=-\frac{2 q+1}{\sqrt{3}}, \quad X_{f_{a L}}=\frac{q-1}{3} .
$$

Then, the electric charge operator, for triplet, has the form

$$
Q=\left(\begin{array}{ccc}
\frac{1}{3}(1-q)+X & \\
& -\frac{1}{3}(2+q)+X & \\
& & \frac{1}{3}(1+2 q)+X
\end{array}\right) .
$$

\section{II.1. Yukawa couplings and fermion masses}

For the leptons

$$
\begin{aligned}
f_{a L} & =\left(v_{a}, l_{a}, E_{a}^{q}\right)_{L}^{T} \sim\left(1,3, \frac{q-1}{3}\right), \\
l_{a R} & \sim(1,1,-1), \quad E_{a R}^{q} \sim(1,1, q) .
\end{aligned}
$$

The mass of $E_{a}^{q}$ is obtained from the Yukawa coupling

$$
-\mathscr{L}_{\text {Yukawa }}^{E}=h_{a b}^{E} \overline{f_{a L}} \Phi_{1} E_{b R}^{q}+\text { h.c. },
$$

where

$$
\Phi_{1} \sim\left(1,3,-\frac{1+2 q}{3}\right)=\left(\begin{array}{c}
\Phi_{1}^{(-q)} \\
\Phi_{1}^{(-q-1)} \\
\Phi_{1}^{0}
\end{array}\right)
$$


Hence, if $\Phi_{1}^{0}$ has a vacuum expectation value (VEV) $\frac{\omega}{\sqrt{2}}$, then $E_{a}^{q}$ gets mass from a mass matrix

$$
\left(m_{E}\right)_{a b}=h_{a b}^{E} \frac{\omega}{\sqrt{2}} .
$$

Finally for the ordinary leptons, we have

$$
-\mathscr{L}_{\text {Yukawa }}^{l}=h_{a b}^{l} \overline{f_{a L}} \Phi_{2} l_{b R}+\text { h.c. },
$$

where

$$
\Phi_{2} \sim\left(1,3, \frac{2+q}{3}\right)=\left(\begin{array}{c}
\Phi_{2}^{(+)} \\
\Phi_{2}^{0} \\
\Phi_{2}^{(q+1)}
\end{array}\right)
$$

If $\Phi_{2}^{0}$ has a $\operatorname{VEV} \frac{v}{\sqrt{2}}$, then the mass matrix related to masses of $l_{a}$ is

$$
\left(m_{l}\right)_{a b}=h_{a b}^{l} \frac{v}{\sqrt{2}} .
$$

We turn now to the quark sector where

$$
\begin{aligned}
Q_{3 L} & =\left(\begin{array}{c}
u_{3} \\
d_{3} \\
T
\end{array}\right)_{L} \sim\left(3,3, \frac{1+q}{3}\right), \\
u_{3 R} & \sim(3,1,2 / 3), \quad d_{3 R} \sim(3,1,-1 / 3), \\
T_{R} & \sim\left(3,1, \frac{2+3 q}{3}\right) .
\end{aligned}
$$

The $u_{3}$ gets mass through the Yukawa part,

$$
-\mathscr{L}_{\text {Yukawa }}^{t}=h^{t} \overline{Q_{3 L}} \Phi_{3} u_{3 R}+\text { h.c. },
$$

where

$$
\Phi_{3} \sim\left(1,3, \frac{q-1}{3}\right)=\left(\begin{array}{c}
\Phi_{3}^{0} \\
\Phi_{3}^{-} \\
\Phi_{3}^{(q)}
\end{array}\right)
$$

If $\Phi_{3}^{0}$ has a VEV $\frac{u}{\sqrt{2}}$ then the mass term of $u_{3}$ is

$$
m_{u_{3}}=h^{t} \frac{u}{\sqrt{2}} .
$$

The other Yukawa terms relating with $Q_{3 L}$ are

$$
-\mathscr{L}_{\text {Yukawa }}^{\mathrm{g} 3}=h^{b} \overline{Q_{3 L}} \Phi_{2} d_{3 R}+h^{T} \overline{Q_{3 L}} \Phi_{1} T_{R}+\text { h.c. },
$$

which give two mass terms:

$$
m_{d_{3}}=h^{b} \frac{v}{\sqrt{2}}, m_{T}=h^{T} \frac{\omega}{\sqrt{2}} .
$$


Two other quark generations are

$$
\begin{aligned}
Q_{\alpha L} & =\left(\begin{array}{c}
d_{\alpha} \\
-u_{\alpha} \\
D_{\alpha}
\end{array}\right)_{L} \sim\left(3,3^{*},-\frac{q}{3}\right), \quad \alpha=1,2 \\
u_{\alpha R} & \sim(3,1,2 / 3), \quad d_{\alpha R} \sim(3,1,-1 / 3) \\
D_{\alpha R} & \sim\left(3,1,-\frac{1+3 q)}{3}\right) .
\end{aligned}
$$

The relevant Yukawa terms are

$$
-\mathscr{L}_{\text {Yukawa }}^{12}=h_{\alpha \beta}^{d 2} \overline{Q_{\alpha L}} \Phi_{3}^{\dagger} d_{\beta R}+h_{\alpha \beta}^{u 2} \overline{Q_{\alpha L}} \Phi_{2}^{\dagger} u_{\beta R}+h_{\alpha \beta}^{D 2} \overline{Q_{\alpha L}} \Phi_{1}^{\dagger} D_{\beta R}+\text { h.c. },
$$

from which it follows

$$
\left(m_{d 2}\right)_{\alpha \beta}=h_{\alpha \beta}^{d 2} \frac{u}{\sqrt{2}}, \quad\left(m_{u 2}\right)_{\alpha \beta}=-h_{\alpha \beta}^{u 2} \frac{v}{\sqrt{2}}, \quad\left(m_{D 2}\right)_{\alpha \beta}=h_{\alpha \beta}^{D 2} \frac{\omega}{\sqrt{2}} .
$$

\section{II.2. Gauge boson masses}

Gauge boson masses arise from the covariant kinetic term of Higgs,

$$
\mathscr{L}_{\text {Higgs }}=\sum_{i=1}^{3}\left(D^{\mu}\left\langle\Phi_{i}\right\rangle\right)^{\dagger} D_{\mu}\left\langle\Phi_{i}\right\rangle
$$

The covariant derivative is defined as

$$
\begin{aligned}
D_{\mu} & =\partial_{\mu}-i g \sum_{a=1}^{8} A_{a \mu} T_{a}-i g^{\prime} X B_{\mu}^{\prime} T_{9} \\
& \equiv \partial_{\mu}-i g P_{\mu}^{N C}-i g P_{\mu}^{C C}
\end{aligned}
$$

where $g, g^{\prime}$ and $A_{a \mu}, B_{\mu}^{\prime}$ are gauge couplings and fields of the gauge groups $S U(3)_{L}$ and $U(1)_{X}$, respectively. For the triplet, $T_{9}=\frac{1}{\sqrt{6}} \operatorname{diag}(1,1,1)$, and the part relating with neutral currents is

$$
\begin{aligned}
P_{\mu}^{N C}=\frac{1}{2} \operatorname{diag} & \left(A_{3}+\frac{A_{8}}{\sqrt{3}}+\sqrt{\frac{2}{3}} X t B^{\prime},-A_{3}+\frac{A_{8}}{\sqrt{3}}+\sqrt{\frac{2}{3}} X t B^{\prime},\right. \\
& \left.-\frac{2 A_{8}}{\sqrt{3}}+\sqrt{\frac{2}{3}} X t B^{\prime}\right)_{\mu},
\end{aligned}
$$

where the space-time indices of gauge fields, for compactness are omitted, and $t \equiv g^{\prime} / g$. The part associated with charged currents is

$$
\begin{aligned}
P_{\mu}^{C C} & =\sum_{a} T_{a} A_{a \mu} ; \quad a=1,2,4,5,6,7 \\
& =\frac{1}{\sqrt{2}}\left(\begin{array}{ccc}
0 & W^{+} & V^{-q} \\
W^{-} & 0 & Y^{-(1+q)} \\
V^{q} & Y^{(1+q)} & 0
\end{array}\right)_{\mu},
\end{aligned}
$$


where we have denoted $\sqrt{2} W_{\mu}^{ \pm} \equiv A_{1 \mu} \mp i A_{2 \mu}, \sqrt{2} V_{\mu}^{ \pm q} \equiv A_{4 \mu} \pm i A_{5 \mu}$ and $\sqrt{2} Y_{\mu}^{ \pm(1+q)} \equiv A_{6 \mu} \pm i A_{7 \mu}$. The upper subscripts label the electric charges of gauge bosons. Remind that this part does not depend on the $X$-charges of triplets.

To summary, with the following Higgs vacuum structure

$$
\left\langle\Phi_{1}\right\rangle=\left(0,0, \frac{\omega}{\sqrt{2}}\right)^{T}, \quad\left\langle\Phi_{2}\right\rangle=\left(0, \frac{v}{\sqrt{2}}, 0\right)^{T}, \quad\left\langle\Phi_{3}\right\rangle=\left(\frac{u}{\sqrt{2}}, 0,0\right)^{T},
$$

masses of non-Hermitian (charged) gauge bosons are given by

$$
m_{W}^{2}=\frac{g^{2}\left(v^{2}+u^{2}\right)}{4}, m_{V}^{2}=\frac{g^{2}\left(u^{2}+\omega^{2}\right)}{4}, m_{Y}^{2}=\frac{g^{2}\left(v^{2}+\omega^{2}\right)}{4} .
$$

By spontaneous symmetry breaking (SSB), the following relation should be in order: $\omega \gg u, v$; and from (26) ones get a consequence

$$
u^{2}+v^{2}=v_{S M}^{2}=246^{2} \quad \mathrm{GeV}^{2} .
$$

The diagonalization of the neutral gauge boson sector is more complicated, because all the three gauge fields generally mix. In the basis $\left(A_{3 \mu}, A_{8 \mu}, B_{\mu}^{\prime}\right)$, the respective squared mass matrix is given by

$$
M_{\text {mass }}^{2 N G}=\frac{g^{2}}{4}\left(\begin{array}{ccc}
u^{2}+v^{2} & \frac{1}{\sqrt{3}}\left(u^{2}-v^{2}\right) & \frac{2 t}{3 \sqrt{6}}\left[(q-1) u^{2}-(q+2) v^{2}\right) \\
& \frac{1}{3}\left(u^{2}+v^{2}+4 \omega^{2}\right) & \frac{2 t}{9 \sqrt{2}}\left[(q-1) u^{2}+(q+2) v^{2}+2(2 q+1) \omega^{2}\right] \\
& \frac{2 t^{2}}{27}\left[(q-1)^{2} u^{2}+(q+2)^{2} v^{2}+(2 q+1)^{2} \omega^{2}\right]
\end{array}\right) .
$$

First of all, we can always obtain a zero eigenvalue (i.e. photon mass) with the corresponding eigenstate (i.e. photon field) as

$$
A_{\mu}=\frac{\sqrt{3} t}{\sqrt{18+4\left(1+q+q^{2}\right) t^{2}}}\left(A_{3 \mu}+b A_{8 \mu}+\frac{\sqrt{6}}{t} B_{\mu}^{\prime}\right),
$$

which is independent of the VEVs as a consequence of the electric charge conservation [3].

Next, we can write electromagnetic interactions following the standard form given in [3], and thus the Weinberg's angle $\left(\theta_{W}\right)$ is defined as

$$
s_{W}=\frac{\sqrt{3} t}{\sqrt{18+4\left(1+q+q^{2}\right) t^{2}}},
$$

where note that $s_{W}=\sin \theta_{W}, c_{W}=\cos \theta_{W}$, and so forth. With this at hand, the photon field is rewritten in terms of

$$
A_{\mu}=s_{W} A_{3 \mu}+c_{W}\left(b t_{W} A_{8 \mu}+\frac{\sqrt{6} t_{W}}{t} B_{\mu}^{\prime}\right) .
$$

The SM $Z$ boson is orthogonal to the photon field as usual,

$$
Z_{\mu}=c_{W} A_{3 \mu}-s_{W}\left(b t_{W} A_{8 \mu}+\frac{\sqrt{6} t_{W}}{t} B_{\mu}^{\prime}\right) .
$$


The model under consideration contains one new, neutral gauge bosons, called $Z^{\prime}$, that are orthogonal to the field in the parentheses (as coupled to the weak hypercharge) appearing in the photon and $Z$ fields, which are obtained by

$$
Z_{\mu}^{\prime}=\frac{1}{\sqrt{6+b^{2} t^{2}}}\left(\sqrt{6} A_{8 \mu}-b t B_{\mu}^{\prime}\right)
$$

where note that $t=\frac{3 \sqrt{2} s_{W}}{\sqrt{3-4\left(1+q+q^{2}\right) s_{W}^{2}}}$.

Next, let us change to the new basis of $\left(A_{\mu}, Z_{\mu}, Z_{\mu}^{\prime}\right)$. Correspondingly, the mass matrix $M_{\text {mass }}^{2 N G}$ is changed to

$$
M^{\prime 2}=U^{T} M_{\mathrm{mass}}^{2 N G} U=\left(\begin{array}{cc}
0 & 0 \\
0 & M^{\prime \prime 2}
\end{array}\right)
$$

where $\left(A_{3 \mu}, A_{8 \mu}, B_{\mu}^{\prime}\right)^{T}=U\left(A_{\mu}, Z_{\mu}, Z_{\mu}^{\prime}\right)^{T}$, and

$$
U=\left(\begin{array}{ccc}
s_{W} & c_{W} & 0 \\
\frac{b t^{2} c_{W}^{2}}{s_{W}\left(6+b^{2} t^{2}\right)} & -\frac{b t^{2} c_{W}}{6+b^{2} t^{2}} & \frac{\sqrt{6}}{\sqrt{6+b^{2} t^{2}}} \\
\frac{\sqrt{6} t c_{W}^{2}}{s_{W}\left(6+b^{2} t^{2}\right)} & -\frac{\sqrt{6} c_{W}}{6+b^{2} t^{2}} & -\frac{b t}{\sqrt{6+b^{2} t^{2}}}
\end{array}\right) .
$$

We see that the photon field, $A_{\mu}$, is decoupled, while the other states $\left(Z_{\mu}, Z_{\mu}^{\prime}\right)$ mix by themselves via a $2 \times 2$ mass matrix, $M^{\prime \prime 2}$, found to be

$$
M^{\prime \prime 2}=\frac{g^{2}}{4}\left(\begin{array}{cc}
\frac{u^{2}+v^{2}}{c_{W}^{2}} & \frac{u^{2}-v^{2}-2\left[(1+q) u^{2}+q v^{2}\right] s_{W}^{2}}{c_{W}^{2} \sqrt{3-4\left(1+q+q^{2}\right) s_{W}^{2}}} \\
\frac{u^{2}-v^{2}-2\left[(1+q) u^{2}+q v^{2}\right] s_{W}^{2}}{c_{W}^{2} \sqrt{3-4\left(1+q+q^{2}\right) s_{W}^{2}}} & \frac{u^{2}+v^{2}+4 \omega^{2}-4 s_{W}^{2}\left[(1+q) u^{2}-q v^{2}+2 \omega^{2}-\left((1+q)^{2} u^{2}+q^{2} v^{2}+\omega^{2}\right) s_{W}^{2}\right]}{c_{W}^{2}\left[3-4\left(1+q+q^{2}\right) s_{W}^{2}\right]}
\end{array}\right) .
$$

Diagonalizing their mass matrix, we obtain the corresponding physical states

$$
Z_{1 \mu}=c_{\varepsilon} Z_{\mu}-s_{\varepsilon} Z_{\mu}^{\prime}, \quad Z_{1 \mu}^{\prime}=s_{\varepsilon} Z_{\mu}+c_{\varepsilon} Z_{\mu}^{\prime},
$$

where the $Z_{\mu}-Z_{\mu}^{\prime}$ mixing angle $(\xi)$ is obtained by

$$
\begin{aligned}
t_{2 \xi}=\tan 2 \xi & =\frac{\sqrt{3-4\left(1+q+q^{2}\right) s_{W}^{2}}\left[v^{2}-u^{2}+2\left((1+q) u^{2}+q v^{2}\right) s_{W}^{2}\right]}{u^{2}+v^{2}-2 \omega^{2}-2 s_{W}^{2}\left[v^{2}+q\left(q u^{2}+(2+q) v^{2}\right)-2 \omega^{2}+\left((1+q)^{2} u^{2}+q^{2} v^{2}+\omega^{2}\right) s_{W}^{2}\right]} \\
& \simeq \frac{\sqrt{3-4\left(1+q+q^{2}\right) s_{W}^{2}}\left[u^{2}-v^{2}-2\left((1+q) u^{2}+q v^{2}\right) s_{W}^{2}\right]}{2 \omega^{2} c_{W}^{4}},
\end{aligned}
$$

and the corresponding masses are

$$
m_{Z_{1}}^{2} \simeq \frac{g^{2}\left(u^{2}+v^{2}\right)}{4 c_{W}^{2}}, \quad m_{Z_{1}^{\prime}}^{2} \simeq \frac{2 g^{2} \omega^{2} c_{W}^{2}}{3-4\left(1+q+q^{2}\right) s_{W}^{2}} .
$$

Because of the condition $u, v \ll w$, the $Z_{1}$ boson has a small mass in the weak scales $(u, v)$ which is identical to the standard model $Z$ boson, whereas the $Z_{1}^{\prime}$ boson is a new, heavy charged gauge boson with mass proportional to $\omega$ scale. The mixing between these two fields is small since $\xi \rightarrow 0$ due to the above condition.

The spontaneous symmetry breaking follows the pattern

$$
S U(3)_{L} \otimes U(1)_{X} \stackrel{\omega}{\longrightarrow} S U(2)_{L} \otimes U(1)_{Y} \stackrel{u, v}{\longrightarrow} U(1)_{Q} .
$$


Corresponding to each step of the breaking, the neutral gauge boson states will be changed as follows:

$$
S U(3)_{L} \otimes U(1)_{X} \stackrel{A_{3}, A_{8}, B^{\prime}}{\longrightarrow} S U(2)_{L} \otimes U(1)_{Y} \stackrel{A_{3}, B, Z^{\prime}}{\longrightarrow} U(1)_{Q}: A, Z, Z^{\prime} .
$$

Now we return to the Higgs content of the model. It is emphasized that if all fermions except neutrinos have the right-handed counterparts, then there need just three Higgs triplets. From the above presentation we explicitly see that: since sum of $X$-charges over three Higgs triplets vanishes, so in the Higgs potential, there always exists a triple Higgs self-coupling $\varepsilon_{i j k} \Phi_{1}^{i} \Phi_{2}^{j} \Phi_{3}^{k}$.

The lepton number operator is constructed from [5]

$$
L=\alpha T_{3}+\beta T_{8}+\mathscr{L} .
$$

For the general case, let us assume that the new extra lepton E acquire lepton number $l$. Applying Eq.(32) to Eq.(2) we obtain

$$
\alpha=0, \beta=\frac{2(1-l)}{\sqrt{3}}, \mathscr{L}_{f_{a L}}=\frac{2+l}{3} .
$$

Hence, lepton number for triplet is in the form

$$
L=\frac{2(1-l)}{\sqrt{3}} \lambda_{8}+\mathscr{L}=\left(\begin{array}{ccc}
\frac{1-l}{3}+\mathscr{L} & \\
& \frac{1-l}{3}+\mathscr{L} & \\
& & \frac{2(l-1)}{3}+\mathscr{L}
\end{array}\right) .
$$

The gauge bosons have the following lepton numbers

$$
L(W)=0, L\left(V^{q}\right)=l-1, L\left(Y^{(1+q)}\right)=l-1 .
$$

To finish this section, we notice that the model presented here is not applicable for the minimal 3-3-1 model [1], where right-handed charged lepton lies in the lepton triplet. Without right-handed lepton singlet, the scalar sextet has to be introduced to provide masses for all leptons. In the recent proposed model called the simplest 3-3-1 model [6], the exotic lepton has the electric charge $\pm 1 / 2 e$. In this case the beta value vanishes.

\section{CONCLUSION}

In this paper, the 3-3-1 model in which exotic leptons has arbitrarily electric charge is presented. We have showed that the spontaneous symmetry breaking in the model requires just three Higgs triplets. Using the general method of diagonalization, the eigenvalues and eigenstates of neutral gauge bosons have been also obtained. We have showed that due to the vanishing of the sum over three $X$ charges of the Higgs triplets, the Higgs potential always has the triple selfcoupling. The lepton number operator of the 3-3-1 model is also presented. The special case with $q= \pm 2$ is interesting and is our future study.

\section{ACKNOWLEDGMENTS}

We thank Dr. L. T. Hue for the general method of diagonalization. This research is funded by Vietnam National Foundation for Science and Technology Development (NAFOSTED) under grant number 103.01-2016.77. 


\section{REFERENCES}

[1] F. Pisano and V. Pleitez, Phys. Rev. D 46 (1992) 410 ; P. H. Frampton, Phys. Rev. Lett. 69 (1992) 2889; R. Foot et al., Phys. Rev. D 47 (1993) 4158.

[2] M. Singer, J. W. F. Valle and J. Schechter, Phys. Rev. D 22 (1980) 738; R. Foot, H. N. Long and Tuan A. Tran, Phys. Rev. D 50 (1994) 34(R) [arXiv:hep-ph/9402243]; J. C. Montero, F. Pisano and V. Pleitez, Phys. Rev. D 47 (1993) 2918; H. N. Long, Phys. Rev. D 54 (1996) 4691; H. N. Long, Phys. Rev. D 53 (1996) 437.

[3] C. A. de S. Pires, O. P. Ravinez, Phys. Rev. D 58 (1998) 035008; A. Doff, F. Pisano, Mod. Phys. Lett. A 14 (1999) 1133; Phys. Rev. D 63 (2001) 097903 ; P. V. Dong, H. N. Long, Int. J. Mod. Phys. A 21 (2006) 6677.

[4] R. A. Diaz, R. Martinez, F. Ochoa, Phys. Rev. D 72 (2005) 035018.

[5] D. Chang and H. N. Long, Phys. Rev. D 73 (2006) 053006.

[6] L. T. Hue, L. D. Ninh, Mod. Phys. Lett. A 31 (2016) 1650062. 